\title{
Systematic Identification and Bioinformatic Analysis of MicroRNAs in Response to Infections of Coxsackievirus A16 and Enterovirus 71
}

\author{
Zheng Zhu, Yuhua Qi, Huan Fan, Lunbiao Cui, and Zhiyang Shi \\ Key Laboratory of Enteric Pathogenic Microbiology, Ministry of Health, Jiangsu Provincial Center for Disease Prevention and Control, \\ No. 172 Jiangsu Road, Nanjing 210009, China \\ Correspondence should be addressed to Zheng Zhu; zhengzhu@jscdc.cn
}

Received 1 August 2016; Accepted 29 September 2016

Academic Editor: Cheng-Feng Qin

Copyright ( 2016 Zheng Zhu et al. This is an open access article distributed under the Creative Commons Attribution License, which permits unrestricted use, distribution, and reproduction in any medium, provided the original work is properly cited.

\begin{abstract}
Hand, foot, and mouth disease (HFMD), mainly caused by coxsackievirus A16 (CVA16) and enterovirus 71 (EV71) infections, remains a serious public health issue with thousands of newly diagnostic cases each year since 2008 in China. The mechanisms underlying viral infection, however, are elusive to date. In the present study, we systematically investigated the host cellular microRNA (miRNA) expression patterns in response to CVA16 and EV71 infections. Through microarray examination, 27 miRNAs (15 upregulated and 12 downregulated) were found to be coassociated with the replication process of two viruses, while the expression levels of 15 and 5 miRNAs were significantly changed in CVA16- and EV71-infected cells, respectively. A great number of target genes of 27 common differentially expressed miRNAs were predicted by combined use of two computational target prediction algorithms, TargetScan and MiRanda. Comprehensive bioinformatic analysis of target genes in GO categories and KEGG pathways indicated the involvement of diverse biological functions and signaling pathways during viral infection. These results provide an overview of the roles of miRNAs in virus-host interaction, which will contribute to further understanding of HFMD pathological mechanisms.
\end{abstract}

\section{Introduction}

Hand, foot, and mouth disease (HFMD) is a common illness among infants and young children, typically characterized by several days of fever, ulcerative vesicles in the oral mucosa, and maculo- or papulovesicular lesions on the hands, feet, and buttocks [1]. Coxsackievirus A16 (CVA16) and enterovirus 71 (EV71), which belong to the genus Enterovirus in the family Picornaviridae, are the two major pathogens causing HFMD. Although most HFMD cases present with mild and self-limiting clinical symptoms, a minority of patients, especially those infected with EV71 virus, rapidly develop severe neurologic complications such as encephalitis, aseptic meningitis, and acute flaccid paralysis, which can lead to pulmonary edema (PE) and even death. Furthermore, these neurologic complications may be also associated with neurologic sequelae, delayed neurodevelopment, and reduced cognitive functioning in children [2]. The outbreaks of HFMD have been reported in many places of the world including the United States [3], Germany [4], Australia [5], Malaysia [6], Taiwan [7], Singapore [8], and Brunei [9]. In the year 2008, large nationwide HFMD epidemic occurred in Mainland China with a substantial morbidity and mortality rate. A national enhanced surveillance system for HFMD has been therefore established in China to facilitate the epidemiological investigation of the disease [10]. There were 1619706,2168737 , and 1828377 cases in the whole country with 509, 567, and 252 deaths in the year of 2011, 2012, and 2013, respectively, reported by National Health and Family Planning Commission of the People's Republic of China. Thus far, no effective vaccines or antiviral drugs are available for HFMD and the molecular mechanisms underlying the infection of CVA16 and EV71 remain elusive. HFMD, therefore, has become a severe public health issue throughout the world.

MicroRNAs (miRNAs) are a class of small, noncoding RNA molecules with a length of 18 25 nucleotides and function as major gene expression regulators at the posttranscriptional level. Generally, these endogenous RNAs specifically 
target the $3^{\prime}$ untranslated regions ( $3^{\prime}$ UTR) of the mRNAs to result in mRNA degradation or translation inhibition based on the degree of sequence complementarity [11]. It has been estimated that more than one-third of all protein-coding genes seem to be miRNA targets in humans by conserved seed pairing [12]. A growing body of evidence has revealed that miRNAs are involved in diverse physiological processes, such as development [13], cell proliferation and differentiation [14, 15], apoptosis [16], and a variety of pathological conditions $[17,18]$. Similarly, recent studies have also focused on the involvement of miRNAs in virus-host interaction networks. Latent infections with some viruses were demonstrated to alter the host cellular miRNA expression patterns, which might be tightly associated with the initiation and progression of diseases $[19,20]$. It has been demonstrated that some cellular miRNAs could directly affect the virus replication. Human liver-specific miR-122 could be utilized to promote viral RNA replication of hepatitis C virus (HCV) [21]. On the other hand, overexpression of miR-30 $\mathrm{e}^{*}$ upregulated IFN- $\beta$ and the downstream IFN-stimulated genes including OAS1, MxA, and IFITM1 to inhibit dengue virus (DENV) replication [22]. Hepatitis $B$ virus (HBV) infection led to the alteration of miRNA expression profile in mouse and human hepatocytes with the upregulation of miR-486-3p, miR-1908, miR-675, and miR-1231, among which miR-1231 was able to suppress HBV replication by targeting core mRNA [23]. Studying miRNA-mediated virus-host interactions might therefore contribute to an elucidation of virus infection mechanism and potential identification of antiviral targets.

Previously there were also several studies reporting the role of miRNAs in enterovirus infection. Tang et al. identified that the expression of host cellular miR-197 was significantly downregulated by EV71 infection and then revealed a novel molecular mechanism that EV71-induced miR-197 downregulation could sustain host RAN protein level to facilitate viral replication [24]. A most recent study characterized the responses of serum miRNA profiles to various EV71 infection diseases and demonstrated that elevated expression of circulating miR-876-5p is a specific marker of severe EV71 infection [25]. Additionally, miR-432* was identified with the ability to stimulate the replication of CVA16 virus in host cells [26]. Although some small RNA molecules were revealed to be involved in the infectious cycle of CVA16 or EV71 by different researchers, no study, to date, was performed to systematically compare the common and differential aspects of cellular miRNA alterations and analyze related biological pathways upon two enteroviral infections. In the present study, we examined the expression profiles of host cellular miRNA in response to CVA16 and EV71 infections and identified miRNAs tightly related to replication process of two viruses. Meanwhile, target gene prediction and subsequent bioinformatic analysis enhanced our understanding of the roles of miRNAs in HFMD pathogenesis.

\section{Materials and Methods}

2.1. Cells and Viruses. Human RD (rhabdomyosarcoma) cells were cultured in Dulbecco's modified Eagle's medium
(DMEM) (Gibco/Invitrogen, Carlsbad, CA) containing 10\% fetal bovine serum (FBS) (Gibco), supplemented with $100 \mathrm{IU} \mathrm{mL}^{-1}$ penicillin $\mathrm{G}$ and $100 \mu \mathrm{g} \mathrm{mL}^{-1}$ streptomycin (Gibco), and incubated at $37^{\circ} \mathrm{C}$ under a humidified atmosphere with $5 \% \mathrm{CO}_{2}$. CVA16 and EV71 strains were isolated from the throat swab specimens of HFMD patients during an outbreak in 2008 in Jiangsu, China. The virus titer was determined by a plaque assay as described previously [27]. The stocks were stored in aliquots at $-70^{\circ} \mathrm{C}$.

2.2. Virus Infection Assay. $\mathrm{RD}$ cells were seeded in 6-well plates at a density of $1 \times 10^{6}$ per well. After overnight incubation, cells were infected with CVA16 or EV71 virus at a multiplicity of infection (MOI) of 1 . After absorption for $1 \mathrm{~h}$ at $37^{\circ} \mathrm{C}$, the cells were washed twice with PBS and covered with DMEM medium containing 2\% FBS for further culture at $37^{\circ} \mathrm{C}$. The infected and control cells were harvested at $6 \mathrm{~h}$ after infection (hpi) and subjected to microarray or quantitative RT-PCR analysis.

2.3. miRNA Microarray. Total RNA extraction was performed from virus-infected cells and mock-infected cells using the Trizol reagent (Invitrogen) according to the manufacturer's instructions. The quality of purified RNA was assessed by agarose gel electrophoresis and spectrophotometer methods. miRNA expression profile was examined based on a microarray assay using a $\mu$ Paraflo $^{\mathrm{TM}}$ microfluidic chip (LC Sciences, Houston, TX, USA) consisting of hybridization probes complementary to all human miRNAs listed in Sanger Institute miRBase Release 17.0. Data derived from the microarray were analyzed by subtracting the background fluorescence and then normalizing the signals using the locally weighted regression (LOWESS) method [28]. The ratios of normalized signals between virus-infected cells and control cells were calculated. Those miRNAs with a $\geq 1.5$-fold change in expression were shown.

2.4. Validation of miRNA Expression Levels by Quantitative $R T$-PCR. miRNAs were isolated from virus-infected cells and control cells using miRNeasy Mini Kit (Qiagen, Germany) and subsequently reverse-transcribed (RT) into cDNA using the One-Step PrimeScript miRNA cDNA Synthesis Kit (TaKaRa, Dalian, China) following the manufacturer's instructions. Real time PCR assays were performed to determine the expression level of each miRNA with the use of SYBR Premix Ex Taq II Kit (TaKaRa). The assay was carried out in a $20 \mu \mathrm{L}$ reaction mixture containing $10 \mu \mathrm{L}$ of 2x SYBR Premix Ex Taq II, $0.4 \mu \mathrm{L}$ of ROX Reference Dye, $0.4 \mu \mathrm{M}$ of specific miRNA forward primer, $0.4 \mu \mathrm{M}$ of UnimiR qPCR Primer (TaKaRa), and $2 \mu \mathrm{L}$ of diluted cDNA (1:50). The thermal cycling conditions were as follows: an initial denaturation step at $95^{\circ} \mathrm{C}$ for $30 \mathrm{~s}, 40$ cycles of PCR amplification at $95^{\circ} \mathrm{C}$ for $5 \mathrm{~s}$, and $60^{\circ} \mathrm{C}$ for $30 \mathrm{~s}$, followed by a melting curve analysis program according to the instrument documentation. All real time PCR reactions were run in triplicate. U6 small RNA was used as an internal control. Fold change of each miRNA expression was calculated using the equation $2^{-\Delta \Delta \mathrm{Ct}}$. The sequences of all the primers used were listed in Table 1. 
TABLE 1: Specific primer sequences for miRNA expression validation by quantitative RT-PCR.

\begin{tabular}{lc}
\hline Primer & Sequence $\left(5^{\prime}-3^{\prime}\right)$ \\
\hline miR-4484 & AAAAGGCGGGAGAAGCCCCAAA \\
miR-4497 & ATTCTCCGGGACGGCTGGGCA \\
miR-4530 & ATTCCCAGCAGGACGGGAGCGAA \\
miR-3665 & ATTAGCAGGTGCGGGGCGGCGAA \\
miR-4455 & CCAGGGTGTGTGTGTTTTTAA \\
miR-4443 & TTGGAGGCGTGGGTTTT \\
U6F & CGCTTCGGCAGCACATATAC \\
U6R & ACGAATTTGCGTGTCATCCT \\
\hline
\end{tabular}

2.5. Target Gene Prediction of Differentially Expressed miRNAs. Two computational target prediction algorithms, TargetScan (http://www.targetscan.org/) and MiRanda (http:// www.microrna.org/), were used to predict the potential targets of the differentially expressed miRNAs, respectively. The obtained data were combined and the target genes predicted simultaneously by both algorithms were presented.

2.6. GO and KEGG Pathway Enrichment Analysis. GO and KEGG pathway enrichment analyses of miRNA target genes were performed as previously described [29]. For GO analysis, all of the target genes were matched to GO terms in the database (ftp://ftp.ncbi.nih.gov/gene/DATA/gene2go.gz) according to three categories: biological process, cellular component, and molecular function, and then the gene numbers for each term were calculated. The significantly enriched GO terms were obtained by comparing with the reference gene background with the use of hypergeometric tests. The calculating formula is as follows:

$$
P=1-\sum_{i=0}^{m-1} \frac{\left(\begin{array}{c}
M \\
i
\end{array}\right)\left(\begin{array}{c}
N-M \\
n-i
\end{array}\right)}{\left(\begin{array}{c}
N \\
n
\end{array}\right)} .
$$

In this formula, $N$ represented the number of GO annotated genes in whole genome, $n$ represented the number of target genes in $N, M$ was the number of single GO annotated genes in whole genome, and $m$ was the number of target genes in $M$. Those GO terms with $P<0.01$ were considered as significant enrichment.

Similarly, all of the target genes of differentially expressed miRNAs were annotated with corresponding KEGG pathways using the database (http://www.genome.jp/kegg/). Enrichment analysis was then performed using the same formula as GO, where $N$ represented the number of KEGG annotated genes in whole genome, $n$ represented the number of target genes in $N, M$ was the number of genes with single KEGG pathway in whole genome, and $m$ was the number of target genes in $M . P<0.01$ was considered as significant enriched KEGG pathway.

\section{Results}

3.1. miRNA Expression Profiles in CVA16- and EV71-Infected Cells. In order to investigate host cellular miRNA expression alterations upon CVA16 and EV71 infections, total RNA was extracted from mock-infected and virus-infected RD cells. Agarose gel electrophoresis analysis showed that the purified total RNA possessed good integrity in three samples (Figure 1(a)). Small RNAs were enriched using a YM-100 Microcon centrifugal filter and then subjected to miRNA microarray analysis. As shown in Figure 1(b), CVA16 and EV71 infections increased the expression levels of 26 cellular miRNAs compared with control cells, among which 15 miRNAs were commonly upregulated by both viruses with 9 CVA16-specific upregulation and 2 EV71-specific upregulation. Meanwhile, the expression levels of 12 miRNAs were decreased simultaneously by both viruses. 6 and 3 miRNAs were specifically downregulated by CVA16 and EV71 infections, respectively. The regulated miRNAs with fold changes were in detail listed in Table 2.

3.2. Validation of miRNA Expression Levels by Quantitative RT-PCR. To confirm the changes of miRNA expression levels induced by CVA16 and EV71 infections, six miRNAs were selected from the miRNA pool regulated by both viruses for expression analysis by qRT-PCR. As shown in Figure 2, miR4484, miR-4497, miR-4530, and miR-3665 showed significant fold increase in expression levels, whereas miR-4455 and miR-4443 were markedly downregulated upon CVA16 and EV71 infections. These results were consistent with those obtained by miRNA microarray analysis.

3.3. miRNA Target Gene Prediction. To further unveil the possible molecular mechanisms underlying HFMD-related virus infection, comprehensive target gene prediction was performed based on the 27 differentially expressed miRNAs (15 upregulated and 12 downregulated, Table 2) from CVA16 and EV17 commonly regulated miRNA pool using the TargetScan and miRanda predicting procedures. Thousands of potential target genes of these 27 miRNAs were successfully predicted simultaneously by both algorithms following the corresponding mapping rules (Supplementary Table S1 in Supplementary Material available online at http://dx.doi.org/10.1155/2016/4302470). Further analysis revealed that each of the differentially expressed miRNAs could target a diversity of genes, while a specific gene could be also targeted by multiple miRNAs. Because immune responses are usually involved in the process of viral infection, many immune-related genes such as CD40 ligand (CD40LG), interleukin 6 receptor (IL-6R), interferon regulatory factor 4 (IRF4), and toll-like receptor 10 (TLR10) were predicted, all of which might participate in host antiviral responses against CVA16 and EV71.

3.4. GO Annotation and Enrichment Analysis. A great number of genes could be probably regulated by CVA16- and EV71-associated miRNAs, whereas the biological characteristics of these genes were largely unknown. We therefore used GO database to perform overall analysis of target gene functions. GO is a standard classified system to describe the molecular function, cellular component, and biological process of a certain gene. The target genes were annotated with corresponding GO terms in Supplementary Table S1 and then subjected to enrichment analysis. The top 5 enriched 
TABLE 2: The differentially expressed miRNAs upon CVA16 and EV71 infections.

\begin{tabular}{|c|c|c|c|c|}
\hline & \multicolumn{2}{|c|}{ Upregulated } & \multicolumn{2}{|c|}{ Downregulated } \\
\hline & miRNA & Fold change & miRNA & Fold change \\
\hline \multirow{15}{*}{ CVA16 and EV71 common } & miR-4484 & $5.47 / 3.99^{*}$ & miR-4455 & $-3.43 /-2.13$ \\
\hline & $\operatorname{miR}-4497$ & $4.38 / 1.54$ & miR-1260b & $-2.72 /-1.84$ \\
\hline & $\operatorname{miR}-4530$ & $4.36 / 2.05$ & miR-4324 & $-2.48 /-2.44$ \\
\hline & miR-1246 & $1.89 / 3.95$ & miR-720 & $-2.28 /-2.07$ \\
\hline & $\operatorname{miR}-494$ & $1.76 / 3.11$ & miR-4534 & $-2.20 /-1.53$ \\
\hline & miR-3665 & $2.94 / 2.02$ & $\operatorname{miR}-107$ & $-2.02 /-1.69$ \\
\hline & miR-3935 & $2.89 / 2.62$ & miR-4443 & $-1.83 /-1.88$ \\
\hline & miR-3591-3p & $1.95 / 2.78$ & miR-320a & $-1.63 /-1.79$ \\
\hline & miR-5096 & $2.40 / 1.82$ & miR-103a & $-1.78 /-1.57$ \\
\hline & $\operatorname{miR}-4277$ & $2.16 / 1.52$ & miR-320c & $-1.56 /-1.65$ \\
\hline & $\operatorname{miR}-4734$ & $2.04 / 1.85$ & $\operatorname{miR}-23 c$ & $-1.61 /-1.54$ \\
\hline & $\operatorname{miR}-19 b$ & $2.00 / 1.70$ & miR-320b & $-1.59 /-1.55$ \\
\hline & miR-181d & $1.56 / 1.87$ & & \\
\hline & miR-30b & $1.64 / 1.83$ & & \\
\hline & $\mathrm{miR}-4687-3 \mathrm{p}$ & $1.64 / 1.66$ & & \\
\hline \multirow{9}{*}{ CVA16-specific } & $\operatorname{miR}-4508$ & 2.99 & miR-4668-5p & -2.49 \\
\hline & miR-638 & 2.95 & let-7b & -1.65 \\
\hline & miR-762 & 2.32 & miR-320d & -1.55 \\
\hline & miR-4466 & 2.16 & miR-151-5p & -1.53 \\
\hline & miR-4787-5p & 2.03 & miR-4739 & -1.51 \\
\hline & miR-3196 & 1.98 & miR-320e & -1.50 \\
\hline & miR-3960 & 1.97 & & \\
\hline & $\mathrm{miR}-4516$ & 1.86 & & \\
\hline & miR-3656 & 1.82 & & \\
\hline \multirow{3}{*}{ EV71-specific } & miR-26b & 1.82 & miR-574-3p & -1.59 \\
\hline & miR-20b & 1.51 & miR-1280 & -1.54 \\
\hline & & & miR-22 & -1.52 \\
\hline
\end{tabular}

${ }^{*}$ The fold change of a miRNA regulated by CVA16/EV71 infection.

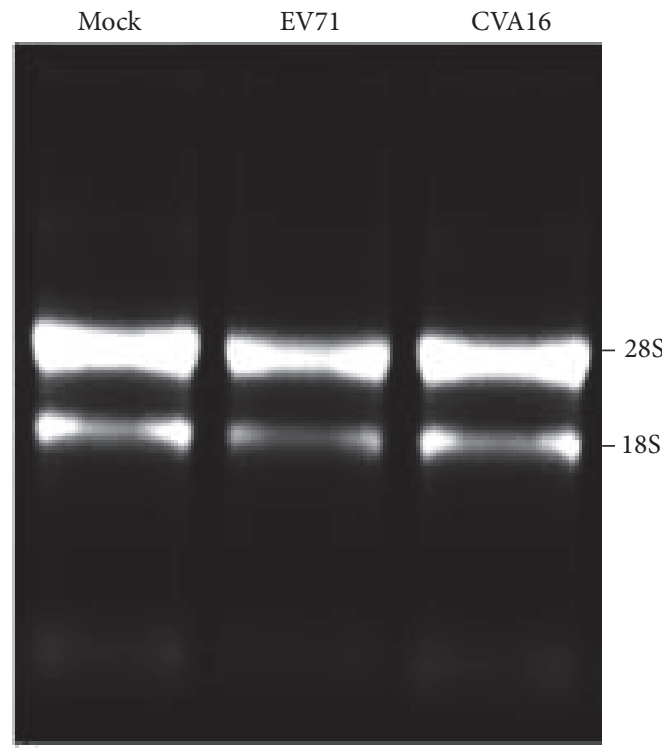

(a)
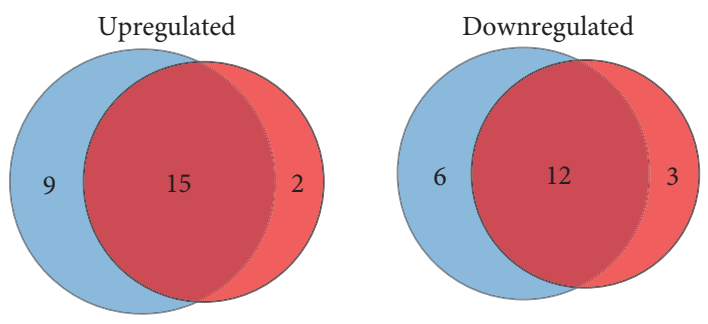

CVA16-infected cells

EV71-infected cells

Figure 1: (a) Agarose gel electrophoresis analysis of total RNA purified from mock-infected and virus-infected RD cells. (b) The alteration of miRNA expression profiles in response to CVA16 and EV71 infection based on microarray analysis. 
TABLE 3: The 5 most enriched terms in each GO category for the target genes of 27 differentially expressed miRNAs.

\begin{tabular}{|c|c|c|c|}
\hline GO ID & GO term & Gene number & $P$ value \\
\hline \multicolumn{4}{|l|}{ Biological process } \\
\hline GO: 0019941 & Modification-dependent protein catabolic process & 292 & $2.12 E-10$ \\
\hline GO: 0007399 & Nervous system development & 259 & $4.10 E-09$ \\
\hline GO: 0006355 & Regulation of transcription, DNA-dependent & 760 & $4.90 E-09$ \\
\hline GO: 0007242 & Intracellular signal transduction & 210 & $1.08 E-07$ \\
\hline GO: 0006468 & Protein phosphorylation & 315 & $1.50 E-06$ \\
\hline \multicolumn{4}{|c|}{ Cellular component } \\
\hline GO: 0005634 & Nucleus & 3367 & $1.23 E-20$ \\
\hline GO: 0005737 & Cytoplasm & 3041 & $1.52 E-17$ \\
\hline GO: 0005794 & Golgi apparatus & 603 & $4.61 E-13$ \\
\hline GO: 0005622 & Intracellular & 1355 & $9.80 E-13$ \\
\hline GO: 0016020 & Membrane & 2561 & $2.50 E-10$ \\
\hline \multicolumn{4}{|l|}{ Molecular function } \\
\hline GO: 0005515 & Protein binding & 3825 & $9.16 E-28$ \\
\hline GO: 0008270 & Zinc ion binding & 1689 & $1.03 E-25$ \\
\hline GO: 0046872 & Metal ion binding & 1759 & $5.63 E-24$ \\
\hline GO: 0003700 & Sequence-specific DNA binding transcription factor activity & 672 & $1.01 E-09$ \\
\hline GO: 0000166 & Nucleotide binding & 1325 & $1.51 E-07$ \\
\hline
\end{tabular}

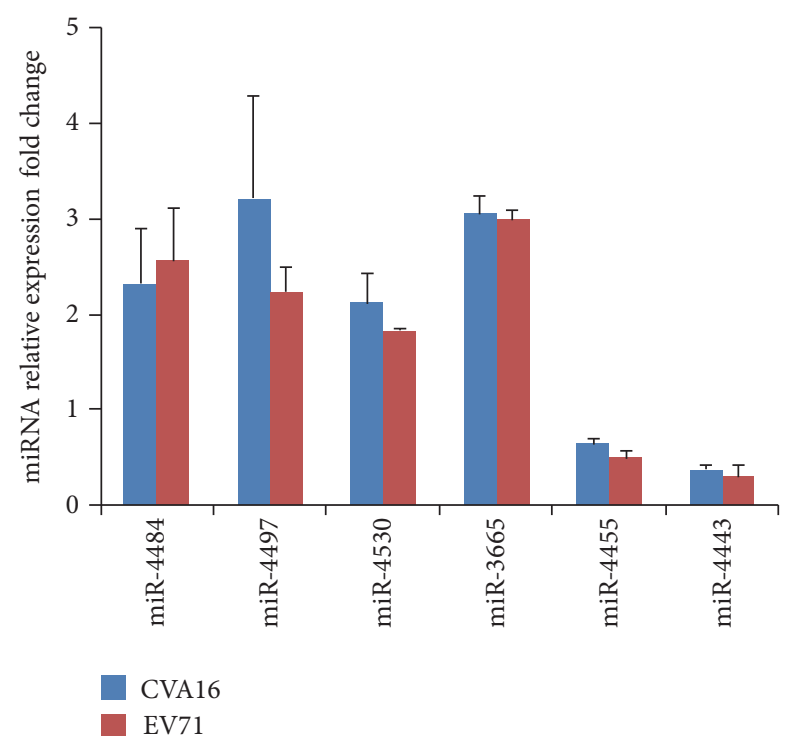

FIGURE 2: Quantitative RT-PCR was performed to examine the expression of miRNAs in virus-infected cells. The expression levels were normalized to endogenous U6 small RNA. Fold change compared with control cells was calculated using the equation $2^{-\Delta \Delta \mathrm{Ct}}$. The data was presented as mean \pm SD. Six miRNAs (miR4484, miR-4497, miR-4530, miR-3665, miR-4455, and miR-4443) exhibited significant fold changes in expression levels $(P<0.05)$.

terms in each GO category for the target genes were listed in Table 3. Meanwhile, the percentage of genes involved in each enriched term was shown in Figure 3.

3.5. KEGG Annotation and Enrichment Analysis. KEGG is a public database to describe the biological pathway in which gene is involved. Similarly, we assigned the miRNA target genes to KEGG pathways (Supplementary Table S1) and found that 54 of the pathways were significantly enriched $(P<0.01$, Table 4 and Supplementary Table S2). More than 150 target genes were related to pathways in cancer (ko05200), MAPK signaling pathway (ko04010), and focal adhesion (ko04510). Wnt signaling pathway (ko04310), TGF-beta signaling pathway (ko04350), and mTOR signaling pathway (ko04150) were also among the enriched pathways of the target genes, reflecting complicated biological events induced by virus infection.

\section{Discussion}

At present, HFMD is still a global public health concern, especially in China. CVA16 and EV71 infections are responsible for most cases of HFMD. The viral pathogenesis, however, remains largely unknown to date. Emerging studies have highlighted the implication of miRNAs as critical effectors in complicated virus-host interaction networks [19-23]. Although sporadic reports revealed the association of a single host miRNA molecule with EV71 infection [24, 25], overall expression alterations of cellular miRNAs upon the infection of HFMD-associated pathogens need to be elucidated. The purpose of our study is to systematically identify the miRNAs involved in CVA16 and EV71 infections as well as investigate their physiological functions by bioinformatic analysis, thus promoting better understanding of HFMD pathological condition.

Microarray assays revealed the miRNA expression profiles in CVA16- and EV71-infected cells. The expression levels of a total of 27 common miRNAs (15 upregulated and 12 downregulated) were found to be significantly changed during the infection process of both viruses, suggesting that these 27 miRNAs might play important roles in the replication 


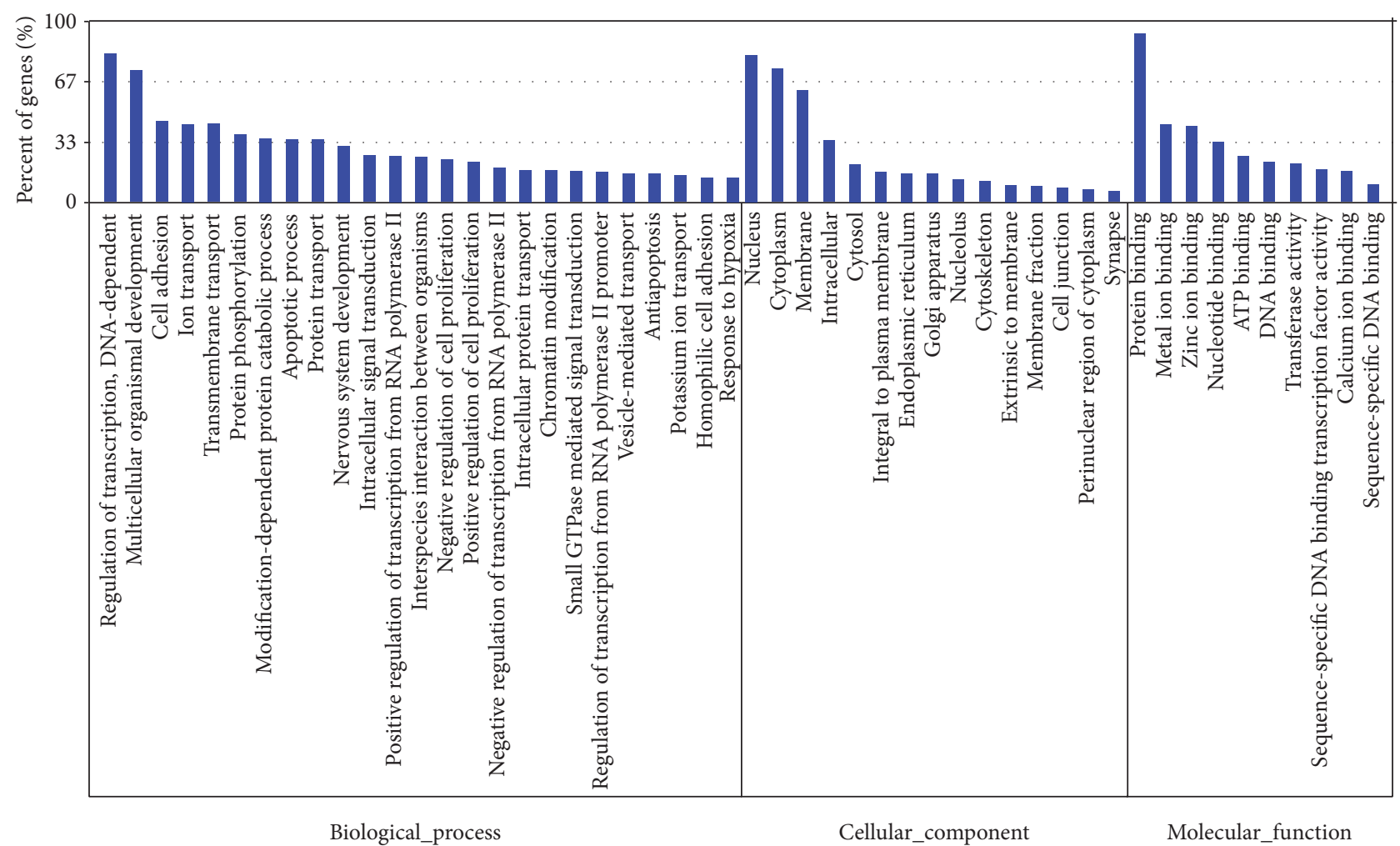

FIgURE 3: The target genes were classified to 3 main GO categories. $Y$-axis represented the percentage of genes in each enriched GO term.

TABLE 4: The 15 KEGG pathways most enriched in the target genes of 27 differentially expressed miRNAs.

\begin{tabular}{|c|c|c|c|}
\hline $\begin{array}{l}\text { Pathway } \\
\text { ID }\end{array}$ & Pathway description & Gene number & $P$ value \\
\hline ko05200 & Pathways in cancer & 256 & $1.18 E-11$ \\
\hline ko04722 & $\begin{array}{l}\text { Neurotrophin signaling } \\
\text { pathway }\end{array}$ & 106 & $3.31 E-09$ \\
\hline ko04012 & ErbB signaling pathway & 75 & $8.40 E-08$ \\
\hline ko04310 & Wnt signaling pathway & 121 & $8.74 E-08$ \\
\hline ko04144 & Endocytosis & 145 & $2.31 E-07$ \\
\hline ko04910 & Insulin signaling pathway & 109 & $7.69 E-07$ \\
\hline ko04510 & Focal adhesion & 153 & $8.55 E-07$ \\
\hline ko05214 & Glioma & 57 & $9.27 E-07$ \\
\hline ko05211 & Renal cell carcinoma & 60 & $2.64 E-06$ \\
\hline ko04720 & Long-term potentiation & 60 & $2.64 E-06$ \\
\hline ko04916 & Melanogenesis & 83 & $3.03 E-06$ \\
\hline ko04010 & MAPK signaling pathway & 199 & $4.31 E-06$ \\
\hline ko04666 & $\begin{array}{c}\text { Fc gamma R-mediated } \\
\text { phagocytosis }\end{array}$ & 79 & $4.93 E-06$ \\
\hline ko04514 & $\begin{array}{l}\text { Cell adhesion molecules } \\
\text { (CAMs) }\end{array}$ & 104 & $1.14 E-05$ \\
\hline ko04070 & $\begin{array}{l}\text { Phosphatidylinositol } \\
\text { signaling system }\end{array}$ & 63 & $1.52 E-05$ \\
\hline
\end{tabular}

course and/or pathogenesis of two enteroviruses. One of the 27 differentially expressed miRNAs identified in our study, miR-1246, was increased by 3.95-fold at the expression level in EV71-infected cells. This finding was consistent with a previous study by $\mathrm{Xu}$ and colleagues [30]. They also found the EV71-mediated upregulation of miR-1246 in infected cells and further unveiled that miR-1246 suppressed the expression of disc-large homolog 3 (DLG3) gene by directly binding the $3^{\prime}$-UTR sequence, which contributed to the neurological disorders induced by EV71 infection. Although the other altered miRNAs have not shown a relationship with the infection of two enteroviruses, some of them seemed to be implicated in molecular mechanisms of host cell interactions with other viruses. For instance, respiratory syncytial virus (RSV), mainly infecting infants and children like HFMDassociated pathogens, induced unique patterns of miRNA expression with specific upregulation of miR-30b in epithelial cells through the NF- $\kappa \mathrm{B}$ signaling pathway, suggesting a possible host defense against RSV by the miRNA response [31]. Similarly, miR-30b was also shown to be tightly associated with chronic HBV and HCV infection [32, 33]. In addition, changes in abundance of circulating miR-320c and miR-494 were also observed in serum of $\mathrm{HCV}$ infected individuals $[34,35]$. At this moment, it is still unknown about the roles of these differentially expressed miRNAs on CVA16 and EV71 viral infection process. Functional studies need to be done with the use of corresponding miRNA mimics or inhibitors in the near future to further investigate their effects on viral replication as well as related molecular mechanisms. Since the 27 miRNAs simultaneously participated in both CVA16 and EV71 interactions with host cells, it is possible that one or two of them could serve as potential candidates for antiviral therapy of HFMD. 
It should be noted that host cells exhibited some differences at the miRNA level in response to CVA16 and EV71 infections, although both viruses belong to members of the genus Enterovirus. CVA16 specifically led to significant expression changes of 15 miRNAs ( 9 upregulated and 6 downregulated), while the expression levels of 5 miRNAs ( 2 upregulated and 3 downregulated) were markedly altered only in EV71-infected cells (Table 2). This may reflect distinct pathological mechanisms underlying the progression of CVA16 or EV71 infection. Indeed, compared with CVA16, acute EV71 infection can cause HFMD frequently followed by serious nervous system diseases, including encephalitis, aseptic meningitis, and acute flaccid paralysis, thus possessing higher mortality. Immunocytochemical staining analysis revealed the existence of viral antigen in neurons, demonstrating the possibility that EV71 actively propagated in nerve cells $[36,37]$. Therefore, it is not surprising that miRNAs may play an essential role in EV71-induced neuropathogenesis. Whether or not the EV71-specific miRNAs (miR-26b, 20b, 574-3p, 1280, and 22) identified in our study are associated with neurological disorders caused by viral invasion will require further investigation. Previous studies have reported that miR-26b could regulate neuronal development and plasticity by direct targeting of brain-derived neurotrophic factor (BDNF) [38]. Overexpression of miR-26b led to aberrant cell cycle entry, tau hyperphosphorylation, and apoptosis in postmitotic neurons, which might contribute to the Alzheimer's disease (AD) neuronal pathology [39]. Meanwhile, miR-22 has been proved to be a potentially neuroprotective miRNA. Its diminished expression was also observed in Huntington's disease (HD) and elevated cellular levels inhibited neurodegeneration in in vitro models through a reduction in caspase activation [40]. These previously elucidated mechanisms will provide guidance for future studies of the involvement of miRNAs in neuropathogenesis caused by EV71 infection.

The EV71-mediated miRNA expression profile identified in present study displayed a little difference from that performed in previous report by $\mathrm{Xu}$ et al. [30]. This discrepancy might be largely due to different cell types used in viral infection between studies. RD cells, as the most sensitive cell line, have been most frequently applied for enteroviral infections in a variety of studies, whereas $\mathrm{Xu}$ and colleagues used SH-SY5Y cells, a human neuroblastoma cell line, to examine the host miRNA response to EV71 infection in nerve cells with the purpose of revealing miRNA-mediated neurological pathogenesis. It is well known that miRNAs have unique tissue cell-specific expression patterns. Each human tissue is characterized by a specific set of miRNAs which may constitute an innate characteristic of that tissue [11, 41]. Moreover, an increasing number of experimental researches have demonstrated that some miRNAs have the potential to modulate the in vivo tissue tropism of multiple viruses [42-44]. So it can be plausible that there is substantial heterogeneity in miRNA expression profile if different tissue cell models were used for the infection of certain pathogen, which depends on further investigation in the near future. Despite this fact, our results confirmed and expanded the findings from a recent study [45] that several cellular miRNA molecules (miR-3665, 4443, 4497, 4530, and 494) were significantly changed not only by EV71 but also by CVA16 viral infection.

In general, mature miRNA population functions as regulators at the posttranscriptional level by directly targeting $3^{\prime}$-UTR regions of mRNAs. Identification of their target genes is therefore a key step to understand the physiological and pathological functions of miRNAs. Target prediction algorithms have been developed as a straightforward and powerful tool to search for the putative miRNA-regulated genes based on seed sequence matching principle. Jointly using two independent target predicting procedures, a great number of target genes of 27 miRNAs coassociated with CVA16 and EV71 infection were obtained. Many of them were related to interleukin, chemokine, interferon, and tolllike receptor, all of which had the ability to mediate host innate and adaptive immune responses against infection of pathogens [46-49]. GO function annotation and enrichment analysis of the target genes according to three categories of biological process, cellular component, and molecular function demonstrated that viral infection might lead to a wide range of regulatory events in host system. Subsequently, KEGG pathway analysis of these putative target genes indicated involvement of Wnt signaling pathway, MAPK signaling pathway, TGF-beta signaling pathway, and mTOR signaling pathway, which have been shown to be important during the infection processes of some other viruses [50-53]. The bioinformatic analysis described above provided a preliminary dataset for additional screening work to characterize the link between specific miRNAs and target genes during host responses to infection of HFMD-associated pathogens.

Taken together, our present study showed that CVA16 and EV71 infections did alter the miRNA expression patterns in host cells with common and specific miRNAs expression changes. Furthermore, systematic bioinformatic analysis provided a comprehensive overview about the relationship between miRNAs and their targets, thus promoting a deeper understanding of viral pathogenesis as well as facilitating the possible development of a miRNA-based therapeutic approach for the prevention and control of HFMD.

\section{Competing Interests}

The authors have no conflict of interests to declare.

\section{Acknowledgments}

This study was supported by Natural Science Foundation of Jiangsu Province (BK20141031) and Jiangsu Province Key Medical Talent Foundation (JKRC2011002).

\section{References}

[1] S. Chea, Y. B. Cheng, K. Chokephaibulkit et al., "Workshop on use of intravenous immunoglobulin in hand, foot and mouth disease in Southeast Asia," Emerging Infectious Diseases, vol. 21, no. 1, Article ID e140992, 2015.

[2] L.-Y. Chang, L.-M. Huang, S. S.-F. Gau et al., "Neurodevelopment and cognition in children after enterovirus 71 infection," The New England Journal of Medicine, vol. 356, no. 12, pp. 12261234, 2007. 
[3] J. P. Alexander Jr., L. Baden, M. A. Pallansch, and L. J. Anderson, "Enterovirus 71 infections and neurologic disease-United States, 1977-1991," Journal of Infectious Diseases, vol. 169, no. 4, pp. 905-908, 1994.

[4] J. Kehle, B. Roth, C. Metzger, A. Pfitzner, and G. Enders, "Molecular characterization of an enterovirus 71 causing neurological disease in Germany," Journal of NeuroVirology, vol. 9, no. 1, pp. 126-128, 2003.

[5] P. McMinn, I. Stratov, L. Nagarajan, and S. Davis, "Neurological manifestations of enterovirus 71 infection in children during an outbreak of hand, foot, and mouth disease in Western Australia," Clinical Infectious Diseases, vol. 32, no. 2, pp. 236242, 2001.

[6] S. AbuBakar, H.-Y. Chee, N. Shafee, K. B. Chua, and S. K. Lam, "Molecular detection of enteroviruses from an outbreak of hand, foot and mouth disease in Malaysia in 1997," Scandinavian Journal of Infectious Diseases, vol. 31, no. 4, pp. 331-335, 1999.

[7] M. Ho, E.-R. Chen, K.-H. Hsu et al., "An epidemic of enterovirus 71 infection in Taiwan. Taiwan Enterovirus Epidemic Working Group," The New England Journal of Medicine, vol. 341, no. 13, pp. 929-935, 1999.

[8] K. Ahmad, "Hand, foot, and mouth disease outbreak reported in Singapore," The Lancet, vol. 356, no. 9238, p. 1338, 2000.

[9] S. AbuBakar, I.-C. Sam, J. Yusof et al., "Enterovirus 71 outbreak, Brunei," Emerging Infectious Diseases, vol. 15, no. 1, pp. 79-82, 2009.

[10] W. Xing, Q. Liao, C. Viboud et al., "Hand, foot, and mouth disease in China, 2008-2012: an epidemiological study," The Lancet Infectious Diseases, vol. 14, no. 4, pp. 308-318, 2014.

[11] D. P. Bartel, "MicroRNAs: genomics, biogenesis, mechanism, and function," Cell, vol. 116, no. 2, pp. 281-297, 2004.

[12] B. P. Lewis, C. B. Burge, and D. P. Bartel, "Conserved seed pairing, often flanked by adenosines, indicates that thousands of human genes are microRNA targets," Cell, vol. 120, no. 1, pp. 15-20, 2005.

[13] A. F. Olena, M. B. Rao, E. J. Thatcher, S.-Y. Wu, and J. G. Patton, " $m i R-216$ a regulates $s n x 5$, a novel notch signaling pathway component, during zebrafish retinal development," Developmental Biology, vol. 400, no. 1, pp. 72-81, 2015.

[14] U. Gioia, V. Di Carlo, P. Caramanica et al., "Mir-23a and mir$125 \mathrm{~b}$ regulate neural stem/progenitor cell proliferation by targeting Musashil," RNA Biology, vol. 11, no. 9, pp. 1105-1112, 2014.

[15] P. Möhnle, S. V. Schütz, V. van der Heide et al., "MicroRNA-146a controls Th1-cell differentiation of human $\mathrm{CD} 4^{+} \mathrm{T}$ lymphocytes by targeting PRKCE," European Journal of Immunology, vol. 45, no. 1, pp. 260-272, 2015.

[16] H.-Q. Zhu, Q. Li, L.-Y. Dong, Q. Zhou, H. Wang, and Y. Wang, "MicroRNA-29b promotes high-fat diet-stimulated endothelial permeability and apoptosis in apoE knock-out mice by downregulating MT1 expression," International Journal of Cardiology, vol. 176, no. 3, pp. 764-770, 2014.

[17] R. Sandhu, J. Rein, M. D’Arcy, J. I. Herschkowitz, K. A. Hoadley, and M. A. Troester, "Overexpression of miR-146a in basal-like breast cancer cells confers enhanced tumorigenic potential in association with altered p53 status," Carcinogenesis, vol. 35, no. 11, pp. 2567-2575, 2014.

[18] G. J. Berry, L. R. Budgeon, T. K. Cooper, N. D. Christensen, and $\mathrm{H}$. Waldner, "The type 1 diabetes resistance locus B10 Idd9.3 mediates impaired B-cell lymphopoiesis and implicates microRNA-34a in diabetes protection," European Journal of Immunology, vol. 44, no. 6, pp. 1716-1727, 2014.
[19] M. Fu, Y. Gao, Q. Zhou et al., "Human cytomegalovirus latent infection alters the expression of cellular and viral microRNA," Gene, vol. 536, no. 2, pp. 272-278, 2014.

[20] A. R. Marquitz, A. Mathur, P. E. Chugh, D. P. Dittmer, and N. Raab-Traub, "Expression profile of microRNAs in Epstein-Barr virus-infected AGS gastric carcinoma cells," Journal of Virology, vol. 88, no. 2, pp. 1389-1393, 2014.

[21] C. L. Jopling, M. Yi, A. M. Lancaster, S. M. Lemon, and P. Sarnow, "Molecular biology: modulation of hepatitis $\mathrm{C}$ virus RNA abundance by a liver-specific MicroRNA," Science, vol. 309, no. 5740, pp. 1577-1581, 2005.

[22] X. Zhu, Z. He, Y. Hu et al., "MicroRNA-30e* suppresses dengue virus replication by promoting NF- $\kappa \mathrm{B}-$ dependent IFN production," PLoS Neglected Tropical Diseases, vol. 8, no. 8, Article ID e3088, 2014.

[23] T. Kohno, M. Tsuge, E. Murakami et al., "Human microRNA hsa-miR-1231 suppresses hepatitis B virus replication by targeting core mRNA," Journal of Viral Hepatitis, vol. 21, no. 9, pp. e89-e97, 2014.

[24] W.-F. Tang, R.-T. Huang, K.-Y. Chien et al., "Host microRNA miR-197 plays a negative regulatory role in the enterovirus 71 infectious cycle by targeting the RAN protein," Journal of Virology, vol. 90, no. 3, pp. 1424-1438, 2016.

[25] R. Y. Wang, K. Weng, Y. Huang, and C. Chen, "Elevated expression of circulating miR876-5p is a specific response to severe EV71 infections," Scientific Reports, vol. 6, p. 24149, 2016.

[26] Z. Yang and P. Tien, "MiR432* regulate the replication of coxsackievirus A16 in rhabdomyosarcoma cells," Wei Sheng Wu Xue Bao, vol. 54, no. 6, pp. 679-687, 2014.

[27] J.-F. Han, R.-Y. Cao, Y.-Q. Deng et al., "Antibody dependent enhancement infection of enterovirus 71 in vitro and in vivo," Virology Journal, vol. 8, article 106, 2011.

[28] B. M. Bolstad, R. A. Irizarry, M. Åstrand, and T. P. Speed, "A comparison of normalization methods for high density oligonucleotide array data based on variance and bias," Bioinformatics, vol. 19, no. 2, pp. 185-193, 2003.

[29] Y. Song, X. An, L. Zhang et al., "Identification and profiling of microRNAs in goat endometrium during embryo implantation," PLoS ONE, vol. 10, no. 4, Article ID e0122202, 2015.

[30] L.-J. Xu, T. Jiang, W. Zhao et al., "Parallel mRNA and microRNA profiling of HEV71-infected human neuroblastoma cells reveal the up-regulation of miR-1246 in association with DLG3 repression," PLoS ONE, vol. 9, no. 4, Article ID e0095272, 2014.

[31] N. J. Thornburg, S. L. Hayward, and J. E. Crowe Jr., "Respiratory syncytial virus regulates human microRNAs by using mechanisms involving beta interferon and NF- $\kappa$ B," $m B i o$, vol. 3 , no. 6 , 2012.

[32] X. Zhang, M. Daucher, D. Armistead, R. Russell, and S. Kottilil, "MicroRNA expression profiling in HCV-infected human hepatoma cells identifies potential anti-viral targets induced by interferon- $\alpha$," PLoS ONE, vol. 8, no. 2, Article ID e55733, 2013.

[33] R. Bruni, C. Marcantonio, A. Pulsoni et al., "microRNA levels in paraffin-embedded indolent B-cell non-Hodgkin lymphoma tissues from patients chronically infected with hepatitis B or C virus," BMC Infectious Diseases, vol. 14, supplement 5, p. S6, 2014.

[34] S. Shwetha, K. Gouthamchandra, M. Chandra, B. Ravishankar, M. N. Khaja, and S. Das, "Circulating miRNA profile in HCV infected serum: novel insight into pathogenesis," Scientific Reports, vol. 3, article 1555, 2013. 
[35] R. El-Diwany, L. N. Wasilewski, K. W. Witwer et al., "Acute hepatitis $\mathrm{C}$ virus infection induces consistent changes in circulating MicroRNAs that are associated with nonlytic hepatocyte release," Journal of Virology, vol. 89, no. 18, pp. 9454-9464, 2015.

[36] C. Hsueh, S.-M. Jung, S.-R. Shih et al., "Acute encephalomyelitis during an outbreak of enterovirus type 71 infection in Taiwan: report of an autopsy case with pathologic, immunofluorescence, and molecular studies," Modern Pathology, vol. 13, no. 11, pp. 1200-1205, 2000.

[37] K. T. Wong, "Emerging and re-emerging epidemic encephalitis: a tale of two viruses," Neuropathology and Applied Neurobiology, vol. 26, no. 4, pp. 313-318, 2000.

[38] V. Caputo, L. Sinibaldi, A. Fiorentino et al., "Brain derived neurotrophic factor (BDNF) expression is regulated by microRNAs miR-26a and miR-26b allele-specific binding," PLoS ONE, vol. 6, no. 12, Article ID e28656, 2011.

[39] S. Absalon, D. M. Kochanek, V. Raghavan, and A. M. Krichevsky, "MiR-26b, upregulated in Alzheimer's disease, activates cell cycle entry, Tau-phosphorylation, and apoptosis in postmitotic neurons," Journal of Neuroscience, vol. 33, no. 37, pp. 1464514659, 2013.

[40] A. Jovicic, J. F. Zaldivar Jolissaint, R. Moser, M. D. F. Silva Santos, and R. Luthi-Carter, "MicroRNA-22 (miR-22) overexpression is neuroprotective via general anti-apoptotic effects and may also target specific Huntington' disease-related mechanisms," PLoS ONE, vol. 8, no. 1, Article ID e54222, 2013.

[41] B. R. Cullen, "Viruses and microRNAs," Nature Genetics, vol. 38, supplement, pp. S25-S30, 2006.

[42] D. Barnes, M. Kunitomi, M. Vignuzzi, K. Saksela, and R. Andino, "Harnessing endogenous miRNAs to control virus tissue tropism as a strategy for developing attenuated virus vaccines," Cell Host and Microbe, vol. 4, no. 3, pp. 239-248, 2008.

[43] K. D. Conrad and M. Niepmann, "The role of microRNAs in hepatitis C virus RNA replication," Archives of Virology, vol. 159, no. 5, pp. 849-862, 2014.

[44] A. V. Kofman, C. Letson, E. Dupart et al., "The p53-microRNA$34 \mathrm{a}$ axis regulates cellular entry receptors for tumor-associated human herpes viruses," Medical Hypotheses, vol. 81, no. 1, pp. 62-67, 2013.

[45] M. Xun, C. F. Ma, Q. L. Du, Y. H. Ji, and J. R. Xu, "Differential expression of miRNAs in enterovirus 71-infected cells," Virology Journal, vol. 12, article 56, 2015.

[46] Q. Wang, X. Chen, J. Feng et al., "Soluble interleukin-6 receptormediated innate immune response to DNA and RNA viruses," Journal of Virology, vol. 87, no. 20, pp. 11244-11254, 2013.

[47] D. Michlmayr, C. S. McKimmie, M. Pingen et al., "Defining the chemokine basis for leukocyte recruitment during viral encephalitis," Journal of Virology, vol. 88, no. 17, pp. 9553-9567, 2014.

[48] C. Yang, X. Zhao, D. Sun et al., "Interferon alpha (IFN $\alpha$ )induced TRIM22 interrupts HCV replication by ubiquitinating NS5A," Cellular and Molecular Immunology, vol. 13, no. 1, pp. 94-102, 2016.

[49] S. M. Y. Lee, K.-H. Kok, M. Jaume et al., "Toll-like receptor 10 is involved in induction of innate immune responses to influenza virus infection," Proceedings of the National Academy of Sciences of the United States of America, vol. 111, no. 10, pp. 3793-3798, 2014.

[50] M. Angelova, K. Zwezdaryk, M. Ferris, B. Shan, C. A. Morris, and D. E. Sullivan, "Human cytomegalovirus infection dysregulates the canonical Wnt/ $\beta$-catenin signaling pathway," PLoS Pathogens, vol. 8, no. 10, Article ID e1002959, 2012.
[51] M. B. Reeves, A. Breidenstein, and T. Compton, "Human cytomegalovirus activation of ERK and myeloid cell leukemia-1 protein correlates with survival of latently infected cells," Proceedings of the National Academy of Sciences of the United States of America, vol. 109, no. 2, pp. 588-593, 2012.

[52] T. Shirasaki, M. Honda, T. Shimakami et al., "Impaired interferon signaling in chronic hepatitis $\mathrm{C}$ patients with advanced fibrosis via the transforming growth factor beta signaling pathway," Hepatology, vol. 60, no. 5, pp. 1519-1530, 2014.

[53] T. Öhman, S. Söderholm, M. Paidikondala, N. Lietzén, S. Matikainen, and T. A. Nyman, "Phosphoproteome characterization reveals that Sendai virus infection activates mTOR signaling in human epithelial cells," Proteomics, vol. 15, no. 12, pp. 2087-2097, 2015. 


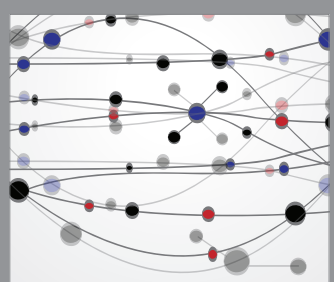

The Scientific World Journal
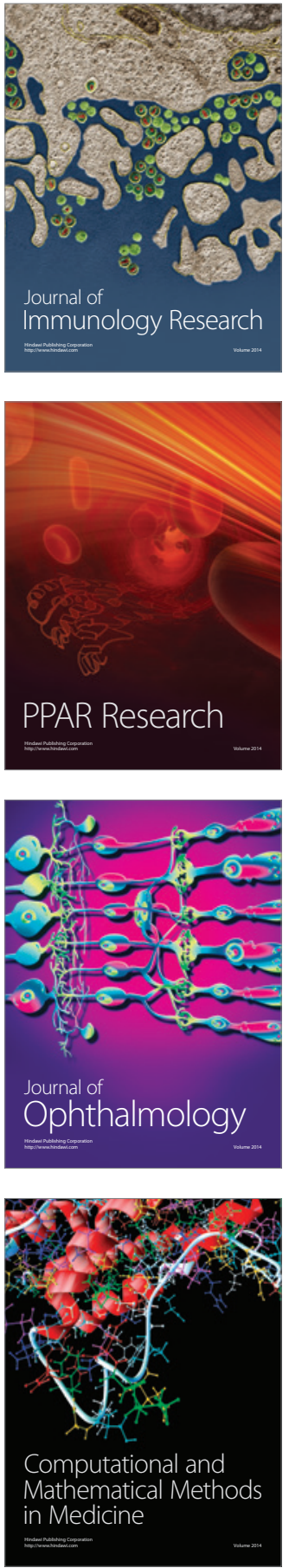

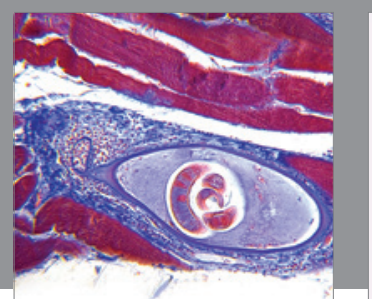

Gastroenterology Research and Practice

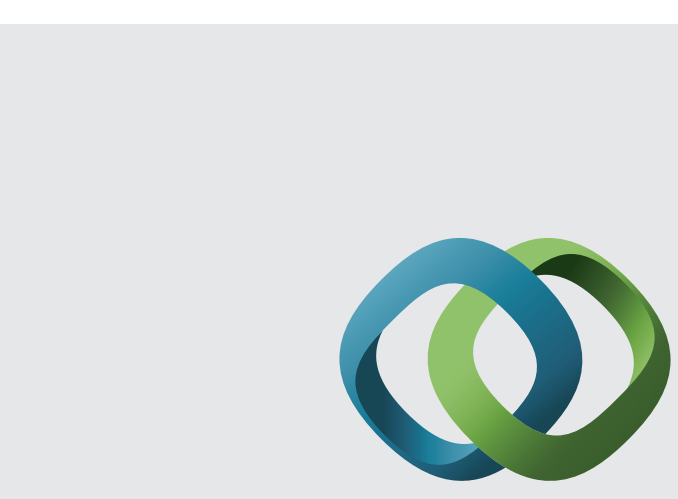

\section{Hindawi}

Submit your manuscripts at

http://www.hindawi.com
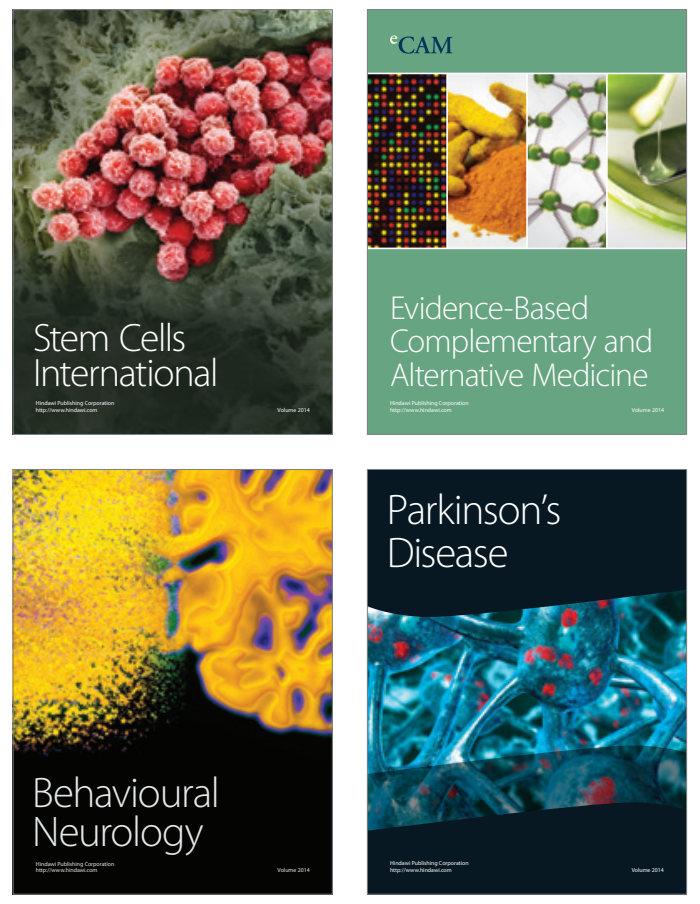
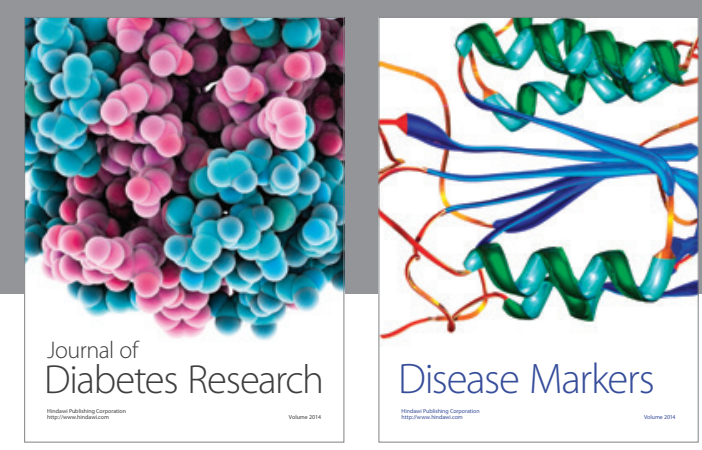

Disease Markers
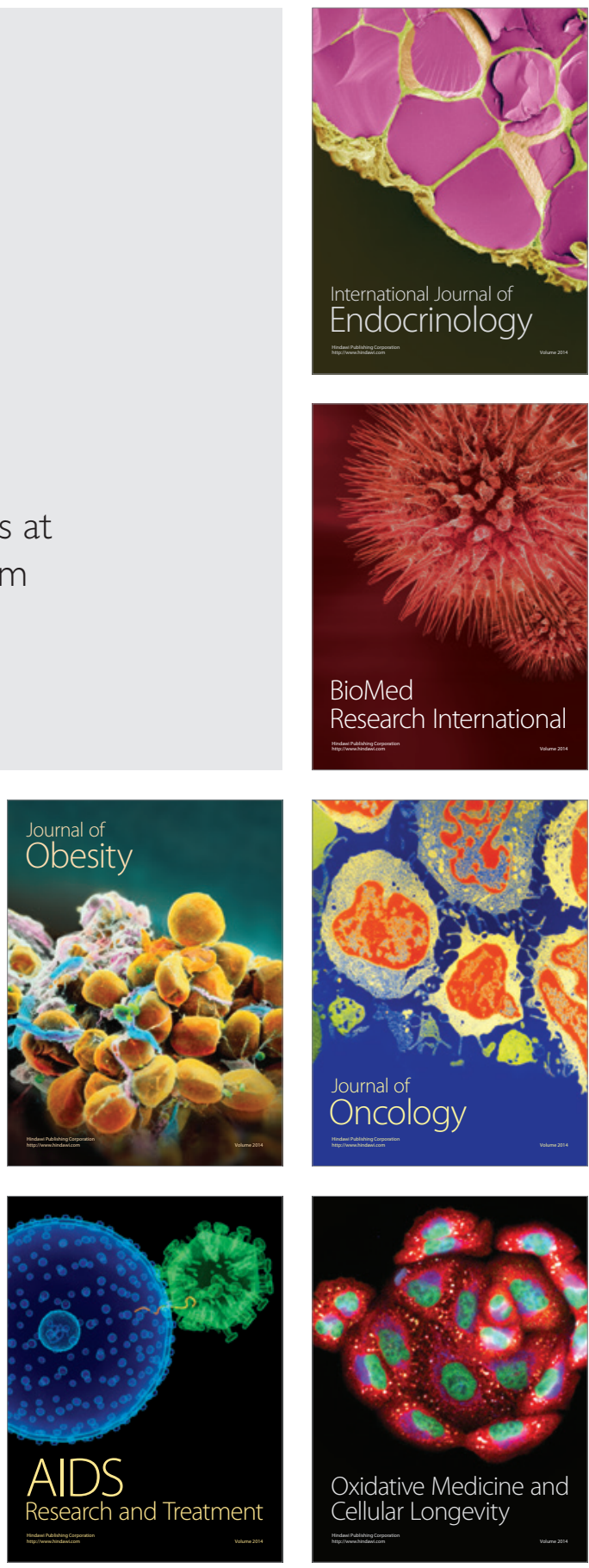